\title{
ADAPTIVE FUSION CONTROL METHOD FOR POWER MOTOR WORKING CONDITION OF NEW ENERGY VEHICLE
}

\author{
Dahai Yu ${ }^{1}$, Yan Gao 1 , Yuanling Liu ${ }^{1}$ \\ ${ }^{1}$ Electric Information College of Changchun Guanghua University, Changchun, 130031, China. \\ Email: yudahaidh00@126.com
}

\begin{abstract}
In order to explore the fusion control strategy of new energy vehicle power motor under different working conditions, in this study, by building a fuzzy control system based on information fusion particle swarm optimization algorithm, the adaptive fusion and control ability of new energy vehicle power motor under different working conditions are studied. Information fusion particle swarm optimization algorithm, fuzzy control system, PID control system in the acceleration, deceleration, stability of three states of motor condition fusion control ability is studied. The research results show that the fuzzy control system based on information fusion particle swarm optimization algorithm is more outstanding than the fuzzy control system and PID control system in terms of the fusion and control ability of electric vehicle motor in stable state, accelerating state and decelerating state. The main performance is that the speed stability of motor is very high, there is basically no obvious fluctuation, the loss of energy is greatly reduced and the efficiency of energy use is improved. It can be seen that the algorithm model of this study has outstanding ability of adaptive fusion control for new energy vehicles. In conclusion, in this study, compared with the common algorithm, the fuzzy control system based on information fusion particle swarm optimization algorithm has obvious advantages for the new energy vehicle motor working condition adaptive fusion control mode, which significantly improves the utilization of energy and reduces the worthless loss. In this study, the fuzzy control system based on information fusion particle swarm optimization algorithm meets the requirements of new energy vehicle electric vehicle for motor adaptive fusion control. The research on the working condition of the power motor of new energy vehicles has a positive effect on the follow-up research.
\end{abstract}

Keywords: Automobile; Working Condition; Motor; Speed of Revolution; Fusion.

\section{Introduction}

With the rapid development of science, technology and economy in recent years, automobile has become a very common means of transportation, and almost every family has at least one car [1].

The rapid development of automobile industry has brought great convenience to people's transportation. However, at the same time, the rapid growth of cars has also brought great pollution to the atmospheric environment, which has seriously threatened the quality of life of the people [2].

Relevant regions have also taken measures such as number limit to control the exhaust emission of vehicles. In the context of this social environment, new energy vehicles come into being [3].

New energy vehicles are mainly divided into two types, pure electric vehicles and hybrid electric vehicles [4].

New energy vehicles rely on power resources as the driving force of the vehicle, without the exhaust emissions brought by traditional vehicles, reducing the pollution of the environment. In addition, new energy vehicles have significant advantages in terms of energy consumption compared with traditional vehicles, not only achieving low pollution, but also achieving low cost [5].

At present, for the actual operation of electric vehicles, there are still some technical difficulties, mainly reflected in the stability of the motor, endurance and the ability to solve complex road conditions [6].

The research on electric vehicles has spread to all levels, and every country has invested huge financial and energy in this field, because new energy vehicles are the most important means of transportation in the future [7].

For the new energy vehicle, the motor is the most core part. The motor is the heart of the new energy vehicle. All working conditions need the analysis and processing of the motor. However, at present, one of the most difficult problems encountered by new energy vehicles in the world is that the motor working condition fusion and control ability are relatively weak under different conditions.

Therefore, improving the integration and control ability of the motor under different conditions is an 
important prerequisite for the stable and safe longterm operation of new energy vehicles [8]. Electric energy plays a very wide role in daily life. Electric power has become the core power of social development, no matter in military, aerospace, industry, life and other aspects. Therefore, it is a very urgent task to accelerate the research on the working condition of motor for the mature landing of new energy vehicles as soon as possible [9].

Only by improving the adaptability to the working condition of the motor can the safety and recognition of new energy vehicles be improved. In this study, by building a fuzzy control system based on information fusion particle swarm optimization algorithm, the motor condition fusion and control status of new energy vehicles are studied, so that the motor condition control effect can be qualitatively and quantitatively analyzed [10].

The internal structure of the motor is very complex, mainly because the motor needs to face a variety of working conditions at all times. If the working conditions can be integrated and coordinated with each other, the energy loss can be minimized and the working efficiency of the motor can be improved. If effective integration and control cannot be achieved, it will reduce the operating power of new energy vehicles. Electric energy is the most stable and efficient clean energy, and its relative value ratio is also relatively high. At present, the research in this field is at a critical stage.

Therefore, the focus of this study is to build and design an effective integrated control system for motor operating conditions [11].

In conclusion, in this study, the adaptive fusion and control ability of the new energy vehicle power motor under different working conditions are studied by building a fuzzy control system based on information fusion particle swarm optimization algorithm. The research shows that the fuzzy control system based on information fusion particle swarm optimization algorithm has obvious advantages over the adaptive fusion control mode of new energy vehicle motor working condition, which significantly improves the utilization of energy and reduces the motor loss. In this study, the algorithm model meets the requirements of the integration control of electric vehicle. The innovation of this study lies in the analysis of the key links of electric vehicle motor working condition. At present, most of the research direction lies in the research of the whole operation system of the electric vehicle. Therefore, this study has a very important value for the follow-up study of the electric vehicle motor condition.

\section{Method Details}

\subsection{Motor and its control technology}

The electric motor is a direct tool to drive the electric vehicle, which is one of the core factors affecting the excellent performance of the vehicle. The motor control flow is shown in Figure 1.

In order to improve the acceleration performance of the car, the driving motor is required to have a large starting torque. If the maximum speed of the car is high, the maximum speed of revolution of the motor is also required to be high. At the same time, it is also required to have the characteristics of small volume, high operation efficiency and high-power generation efficiency as a generator [12].

In the electric drive system, the motor can be used to realize the mutual conversion of electric energy and mechanical energy. The motor consists of rotor and stator [13].

The permanent magnet motor includes a rotor which includes a series of magnets and a current carrying stator separated by air gap. There are three types of motors available for hybrid or plug-in electric vehicle traction drive systems [14].

The built-in permanent magnet motor has high power density and can maintain high efficiency within a very high working limit.

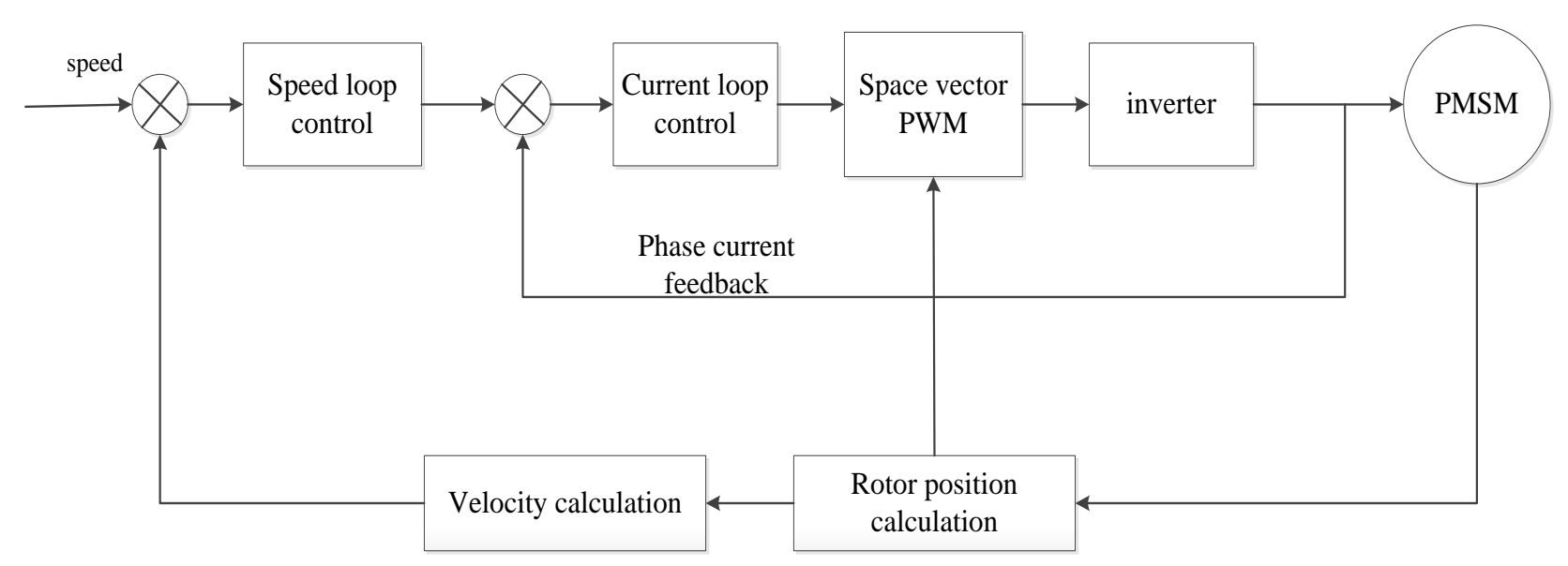

Figure 1: Motor control process 


\subsection{Motor conditions of new energy vehicles}

New energy vehicles rely on the motor very highly, not only to ensure the normal driving of the car, but also to ensure the integration of working conditions. Therefore, compared with the motor of ordinary vehicles, the motor of new energy vehicles has better technical requirements, mainly in the following aspects:

Weight and volume: the motor of new energy vehicles has strict requirements. The weight and volume should be as small as possible, and meet

the design requirements of new energy motors [15]. Under the condition of meeting the weight and volume, the motor has very high requirements for mechanical strength. It is necessary to use light materials as much as possible to reduce the weight of the motor, and gather the reduction positions in one place as much as possible [16].
High efficiency: the high efficiency of the motor is very important for the normal and stable operation of the motor. New energy vehicles will face a variety of complex road conditions in the operation process, and the integration of motor conditions is bound to be affected [17]. High efficiency motor can save energy to the maximum extent and reduce unnecessary loss. Therefore, to ensure the efficiency of the motor is very important for the safe and stable driving of new energy vehicles.

Reliability: reliability is a very important parameter in motor evaluation index. Without the reliability of the motor, the new energy vehicle cannot really achieve efficient driving. Without the reliability and stability of the motor, the integration of various working conditions and the control coordination ability of the motor will be greatly affected. The structure of fuzzy controller is shown in Figure 2.

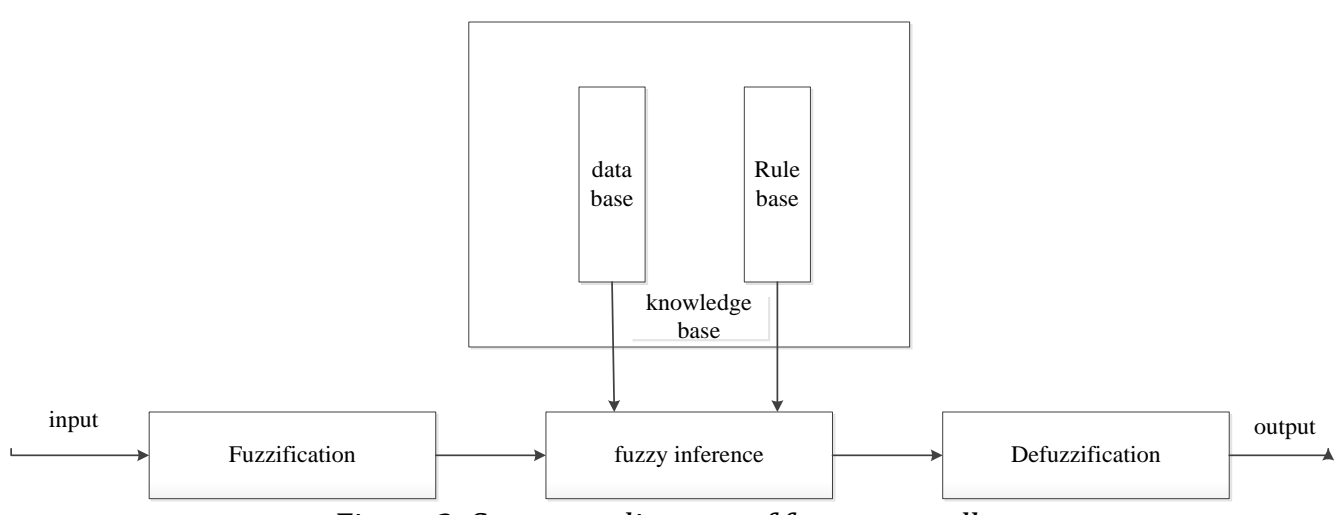

Figure 2: Structure diagram of fuzzy controller

Voltage: for new energy vehicles, quality is an indicator that needs to be strictly controlled, so the quality of the motor must be small. However, due to the need to meet the long-term operation of new energy vehicles, the voltage of the motor must be very high to meet the energy required by the vehicle operation. On the one hand, it can reduce the cost of motor. On the other hand, the overall layout and efficiency can be improved.

Torque and power: torque is the characteristics of the motor itself. Even without the assistance of the transmission, the torque can also achieve the speed regulation of the motor, which can achieve the torque required in different conditions such as deceleration, acceleration, stability, etc. Power refers to the output efficiency of the motor. By controlling the power, the energy efficiency can be maximized under different working conditions.

\subsection{Dynamic model of new energy vehicle}

Like traditional fuel vehicles, electric vehicles are subject to four main resistances when driving on the road: rolling resistance, air resistance, slope resistance and acceleration resistance.
The total resistance to driving is:

$$
\mathrm{F}=\mathrm{F}_{\mathrm{f}}+\mathrm{F}_{\omega}+\mathrm{F}_{\mathrm{i}}+\mathrm{F}_{\mathrm{j}}
$$

Among them, rolling resistance is:

$$
\mathrm{F}_{\mathrm{f}}=\mathrm{fmg} \cos \alpha
$$

Air resistance is:

$$
\mathrm{F}_{\mathrm{w}}=\frac{\mathrm{C}_{\mathrm{d}} \mathrm{Av}^{2}}{21.15}
$$

Slope resistance is:

$$
\mathrm{F}_{\mathrm{i}}=\operatorname{mg} \sin \alpha
$$

Acceleration resistance is:

$$
\mathrm{F}_{\mathrm{j}}=\delta \mathrm{m} \frac{\mathrm{dv}}{\mathrm{dt}}
$$

The relationship between vehicle speed and speed of revolution is as follows:

$$
\mathrm{F}_{\mathrm{j}}=0.377 \frac{\mathrm{nr}}{\mathrm{i}_{0} \mathrm{i}_{\mathrm{g}}}
$$

The torque equation is:

$$
\mathrm{T}=\mathrm{Fr}
$$


The motor load torque is:

$$
\mathrm{T}_{\mathrm{L}}=\frac{\mathrm{T}}{\eta \mathrm{I}_{0} \mathrm{I}_{\mathrm{g}}}
$$

The power performance of the whole vehicle is not only limited by the driving force of the motor, but also restricted by the ground adhesion conditions. The design of driving wheel shall meet the following requirements:

$$
\left\{\begin{array}{l}
\mathrm{F}_{\mathrm{X}}-\mathrm{F}_{\varphi}=\varphi \mathrm{F}_{\mathrm{Z}} \\
\mathrm{F}_{\mathrm{Xmax}}=\varphi \mathrm{F}_{\mathrm{Z}}
\end{array}\right.
$$

Front wheel drive vehicles shall meet the following requirements:

$$
\frac{\mathrm{T}_{\mathrm{t}}-\mathrm{T}_{\mathrm{f} 1}}{\mathrm{r}}=\mathrm{F}_{\mathrm{X} 1} \leq \mathrm{fF}_{\mathrm{Z}}
$$

The moment for the front and rear wheel grounding points is taken, and it can be obtained that:

$$
\left\{\begin{array}{l}
F_{Z 1} L=G b+m \frac{d v}{d t} h_{g} \\
F_{Z 2} L=G a-m \frac{d v}{d t} h_{g}
\end{array}\right.
$$

If $\mathrm{Z}$ is the braking strength, it can be obtained that:

$$
\left\{\begin{array}{l}
F_{\mathrm{Z} 1}=\frac{G}{L}\left(b+z h_{g}\right) \\
F_{\mathrm{Z} 2}=\frac{G}{L}\left(a-z h_{g}\right)
\end{array}\right.
$$

The safety of vehicle braking is affected by the distribution of front and rear brake force. In the process of braking, there may be three working conditions: rear wheel, front wheel or front and rear wheel locking at the same time. Among them, compared with the former two conditions, the vehicle's directional stability and adhesion utilization ratio are better when the front and rear wheels are locked at the same time. At this point, there are:

$$
\begin{aligned}
& \left\{\begin{array}{c}
F_{\mu 1}+F_{\mu 1}=\varphi G \\
F_{\mu 1}=\varphi F_{Z 1} \\
F_{\mu 2}=\varphi F_{Z 2}
\end{array}\right. \\
& \left\{\begin{array}{c}
F_{\mu 1}+F_{\mu 1}=\varphi G \\
\frac{F_{\mu 1}}{F_{\mu 2}}=\frac{F_{Z 1}}{F_{Z 2}}=\frac{b+\varphi h_{g}}{a-\varphi h_{g}}
\end{array}\right.
\end{aligned}
$$

Eliminating $\varphi$, it can be obtained that:

$$
F_{\mu 2}=\frac{1}{2}\left[\begin{array}{l}
\frac{G}{h_{g}} \sqrt{b^{2}+\frac{4 h_{g} L}{G} F_{\mu 1}} \\
-\left(\frac{G}{h_{g}}+2 F_{\mu 1}\right)
\end{array}\right]
$$

\subsection{Improved particle swarm optimization}

In this study, combined with the speed control requirements of electric vehicles, the particle swarm optimization algorithm is improved. For inertia weight, the method of exponential decreasing is adopted. The formula is as follows:

$$
\mathrm{W}=\mathrm{W}_{\max }\left(\frac{\mathrm{W}_{\min }}{\mathrm{W}_{\max }}\right)^{1 /\left(1+10 \mathrm{k} / \mathrm{k}_{\text {max }}\right)}
$$

In the initial stage of optimization, $w$ is large and has strong ability of searching in a large range. In the later stage of optimization, $w$ is smaller and the ability of searching in small range is stronger, which improves the performance of particle swarm optimization. At the same time, in order to avoid the premature convergence of the algorithm, the learning factor is dynamically adjusted. The equation is as follows:

$$
\begin{aligned}
& \mathrm{c}_{1}=2-\sin \frac{\mathrm{k} \pi}{\mathrm{k}_{\text {max }}} \\
& \mathrm{c}_{2}=1+\sin \frac{\mathrm{k} \pi}{\mathrm{k}_{\max }}
\end{aligned}
$$

In the early stage of population search, c1 is larger and c2 is smaller, which is convenient for particles to learn their own optimal solution and improves the global search ability. In the later stage of population search, c2 is larger and c1 is smaller, which is convenient for the population to approach the global optimal solution and enhances the local optimization performance.

Parameter optimization flow of particle swarm optimization is shown in Figure 3. The steps of parameter optimization are as follows. (1) Particle swarm optimization algorithm initialization: the parameters to be optimized are quantization factor and scale factor, so the number of optimization dimensions is 5. (2) Fitness calculation: fitness is the performance evaluation index of particles in particle swarm optimization algorithm, so as to optimize the quantization factor and scale factor. (3) Update and stop of Particle Swarm: particle swarm outputs fitness results through SIMULINK model, and then transmits them to particle swarm optimization algorithm for calculation. When the set evaluation index or the maximum number of iterations is reached, the algorithm is terminated. Finally, the optimized quantization factor and scale factor are input into the simulation model of fuzzy control. 


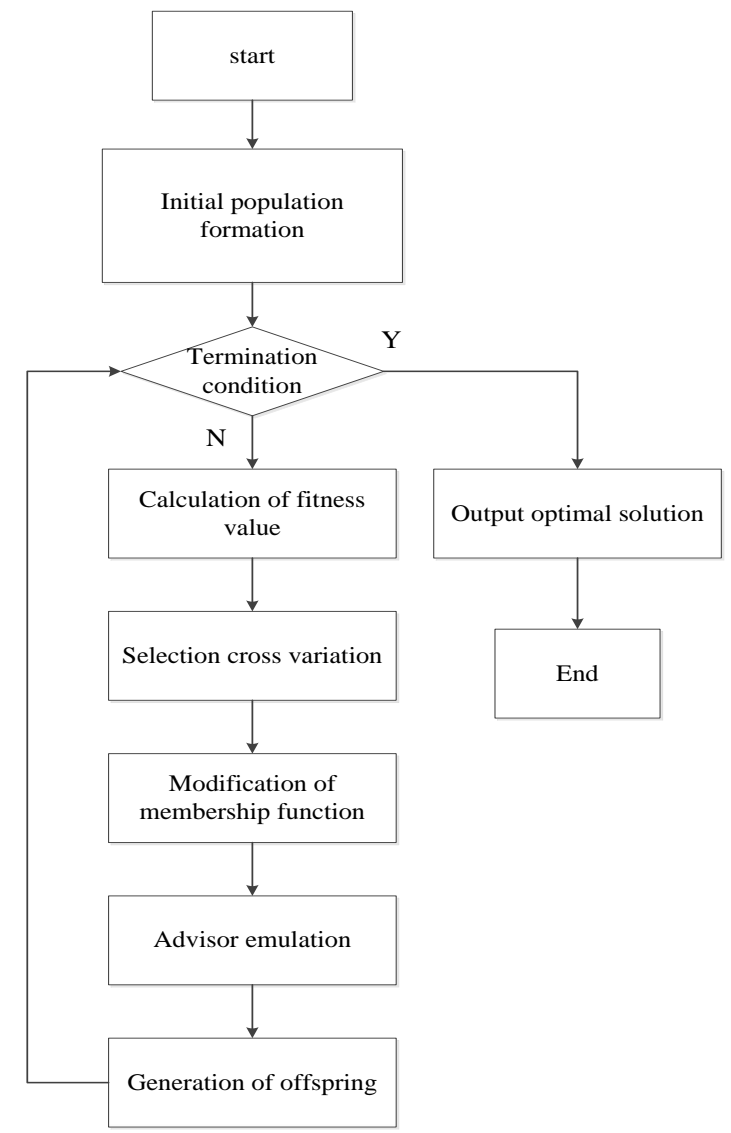

Figure 3: Parameter optimization flow of particle swarm optimization

\subsection{Information fusion technology}

Information fusion technology can be regarded as a technology that borrows computer as carrier and relies on sensor as means to obtain information and data. Through sensors or other acquisition methods, the full range of data of the target is obtained.

Through processing, screening, analysis and other means, useful information is extracted. In the information fusion system, the feedback to determine the target information is multi-angle and multi-level, such as: humidity, temperature, height, $\mathrm{pH}$ and other information, and the way to obtain the valuable information and data of the target is also diverse, such as a variety of sensors and so on.

Through the development of Internet computer technology and related science, at present, it has formed a lot of effective algorithms. According to the requirements, the algorithm can be adjusted in different angles and directions to meet the needs of the design. Through the collaborative operation of algorithms, a lot of obtained information can be analyzed and studied, so as to get the desired results.

The structure of fuzzy control system is shown in Figure 4. Therefore, a perfect information fusion system needs coordination and cooperation from many aspects.

The key link is still how to obtain the most comprehensive and accurate information.

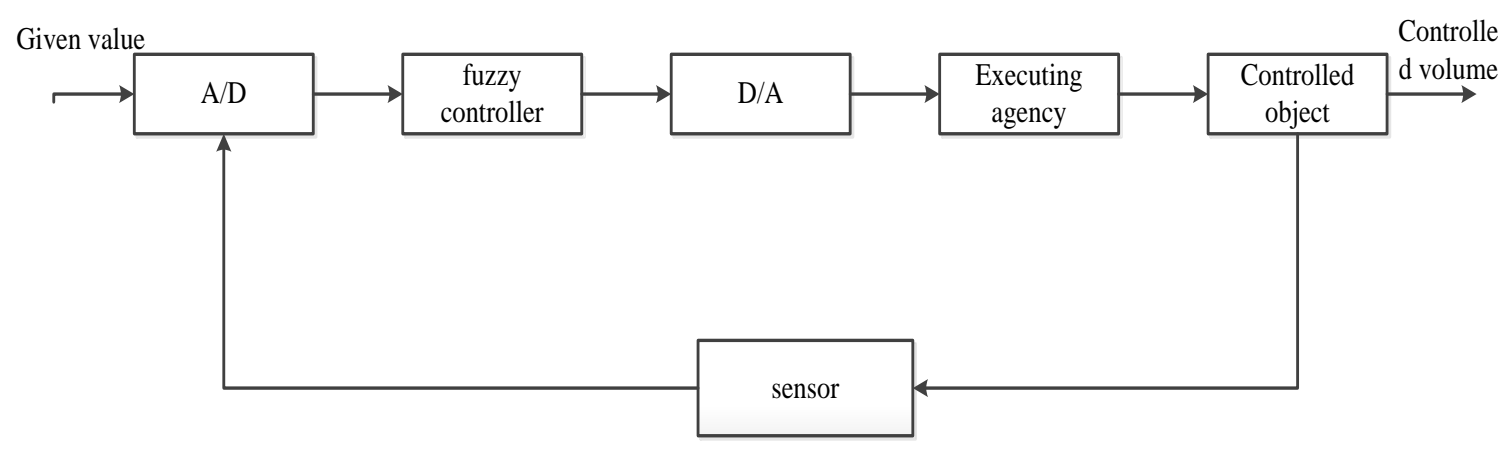

Figure 4: Structure diagram of fuzzy control system

\subsection{Information fusion features}

Stability: because the principle of information fusion technology is to rely on sensors and multidirectional channels to obtain effective information, the most prominent part of its stability is that there is no interference between the ways of obtaining information, and they are independent of each other. Therefore, even if the way of obtaining information from individual channels fails, it will not have a large-scale impact on the overall situation.

This design greatly improves the efficiency and stability of information system.

Spatial resolution: information fusion technology relies on multi-sensor access to information.
Compared with the working mode of a single sensor, this working mode has a significant improvement in spatial resolution.

Conclusion: depending on the way of multichannel and multi-sensor access to information, the information fusion system can monitor the target repeatedly and test each other for the acquired target information, which greatly reduces the probability of error information. In this way, not only the accuracy of the results is improved, but also the working performance of the system is significantly improved.

More information: the biggest difference between single sensor and multi-sensor in acquiring target information is that multi-sensor can monitor the target in all directions without dead angle and obtain 
rich multi-level information. The performance of a single sensor is relatively single, the efficiency is low, and the information cannot be mutually checked. Multi-sensor can remove this disadvantage comprehensively.

At the same time, because the information fusion technology has relatively stable and comprehensive characteristics, the judgment accuracy of multisensor system has significantly improved and unnecessary risks have been reduced.

\subsection{The principle of information fusion}

Information fusion is to obtain valuable information from incomplete or inaccurate information in a specific way. The principle of information fusion makes the best use of its channels and ways under specific rules and requirements, such as sensors or other acquisition methods, to obtain the full range of data of the target, and to extract practical information through processing, screening, analysis and other means.

Information fusion is essentially a process of simulating human brain to deal with comprehensive and complex problems. Human and many creatures have the basic function of multi-sensor information fusion. Human beings detect the surrounding environment information through perceptual organs, and make a comprehensive judgment based on prior knowledge to estimate the surrounding environment and state of affairs. To deal with this complex process, human beings are adaptive. In this process, the external information obtained by human senses is finally integrated into the effective information of observation target recognition.

In this process, a lot of data processing is required, and at the same time, a wealth of prior knowledge is also needed to interpret the comprehensive information obtained.

The principle of PID control system is shown in Figure 5.

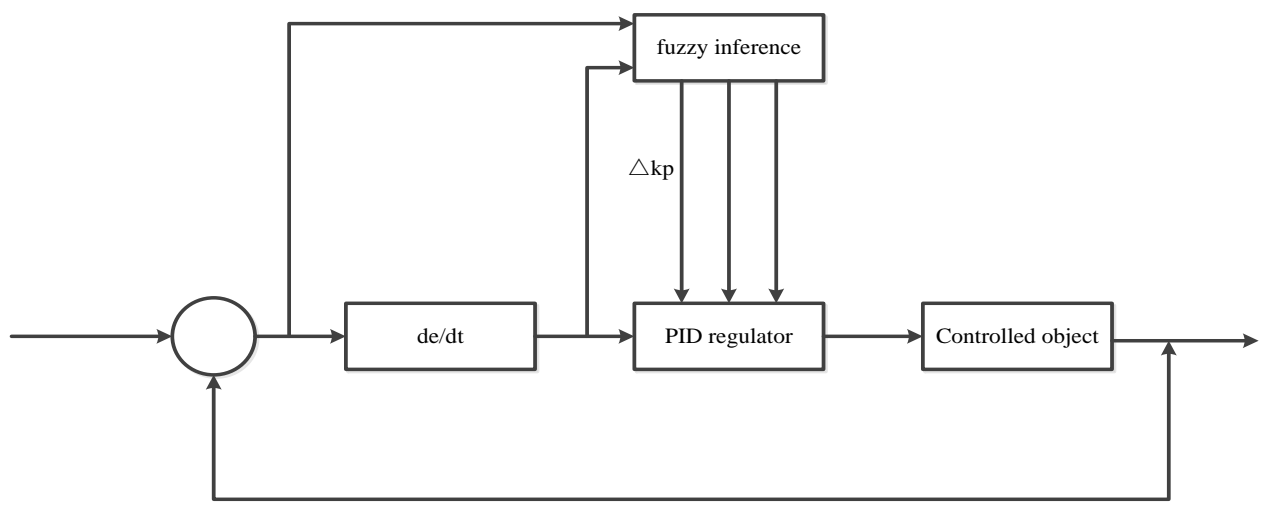

Figure 5: PID control system schematic diagram

The information fusion technology is used to analyze and study the acquired information according to the corresponding rules, evaluate and process the acquired information comprehensively, so as to obtain a more objective, comprehensive and accurate evaluation of the target things. Generally speaking, information fusion technology has 8 system modules, including structural decision subsystem, optimization module, technical analysis system, information optimization system, decision optimization module, decision accuracy subsystem, association system and information counter matching module.

\subsection{Motor drive control strategy}

In general, motor control speed of new energy vehicles is mainly controlled by voltage, speed of revolution, torque and other links.

Voltage control strategy: Generally speaking, the motor power of electric vehicles is large, up to tens of kilowatts. In order to make the drive motor have high output power and work efficiency, it is required that the armature current of the motor is also relatively large, reaching thousands of amperes. At the same time, the demand of armature resistance is very small to meet the demand.

When the flux of the motor is fixed, the speed and electromotive force of the motor will be larger, so the slope of the motor regulation characteristic curve will be larger. When the motor works, its speed and battery power supply voltage will change continuously, which is called stepless motor speed regulation. Compared with other adjustment methods, stepless speed regulation method is relatively smooth. When the control voltage of the power battery changes, under the condition of voltage control, the accelerator pedal can respond to the control signal of motor voltage change. When the hardness of the mechanical characteristics of the motor is certain, and the motor is running at low speed, the voltage is reduced.

At this time, the speed of the motor changes less with the load, so the stability of the motor is better.

Speed strategy: compared with traditional vehicles, new energy vehicles have very different 
motor speed. In the face of different road conditions and different resistance, the traditional automobile realizes the corresponding control of motor speed through the control of throttle, which is the maximum speed that the motor can achieve when facing the corresponding conditions.

New energy vehicles can achieve the optimal output of energy speed under corresponding conditions, which not only meets the load demand but also saves energy.

Motor output torque control strategy: for the current electric vehicles, the permanent magnet brushless DC motor is the ideal driving motor choice. Generally, the traditional linear PID controller is used to control the motor, but the negative feedback current control is needed. This strategy is a way of torque control, which belongs to torque control strategy in essence. At the same time, by changing the input current to complete the control of the motor, the torque control must be realized by using the role of the sensor. In this process, Hall current sensor is needed to detect the current of motor armature. The principle of PID control system is shown in Figure 6.

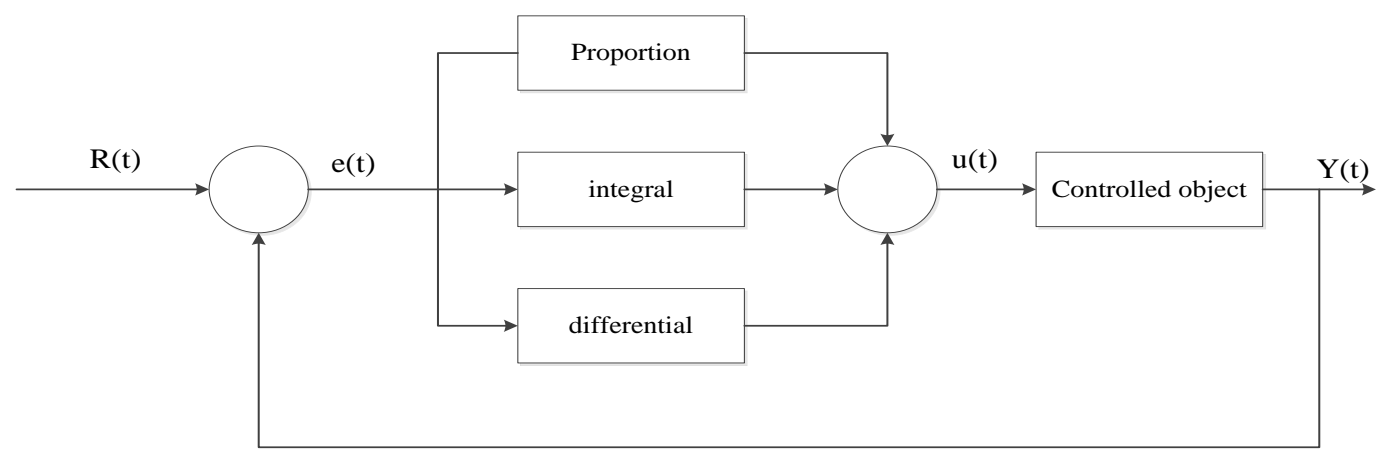

Figure 6: PID control system schematic diagram

Selection of motor control strategy: at present, in the normal speed range, the output force moment of traditional internal combustion engine is approximately constant force moment. By adjusting the driving speed of the vehicle by driver, the closedloop speed control of the motor can be realized. If the driver is regarded as one of the control links, a closed-loop speed control system with driver's participation will be formed. The inner part is a double closed-loop control system with armature current closed-loop control.

This control strategy is more suitable for manually operated vehicles.

\section{Results and Discussion}

When the working condition of power motor of new energy vehicle is stable, the speed comparison curve is shown in Figure 7. From the data and trend chart in the figure, it can be seen that with the passage of time, the three different algorithms have different fusion control capabilities for the motor speed under stable working conditions.

In this study, the fuzzy control system based on information fusion particle swarm optimization shows outstanding control and fusion ability for motor condition fusion control, which mainly shows that the speed is basically stable without significant fluctuation.

However, the adaptability of fuzzy control system and PID control system to motor stability is poor, which leads to the long-term fluctuation of motor speed. It can be seen that the fuzzy control system based on information fusion particle swarm optimization algorithm has outstanding ability of fusion and control for electric vehicle in stable state.

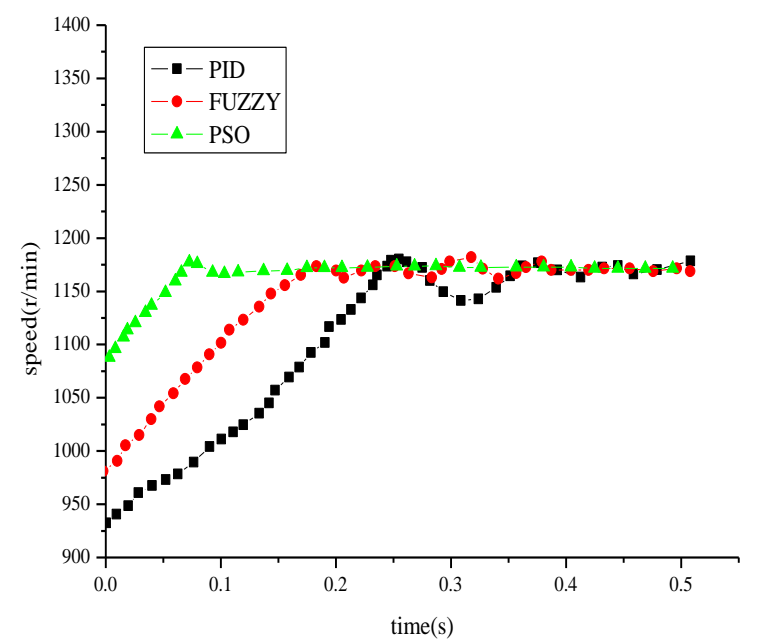

Figure 7: Speed comparison curve of power motor of new energy vehicle under stable working condition

The speed comparison curve of power motor of new energy vehicle under acceleration condition is shown in Figure 8.

From the data and trend chart in the figure, it can be seen that with the change of time, the speed of electric vehicle motor will gradually stabilize under the condition of acceleration.

In this study, three kinds of algorithm structures are used to study the motor working condition in acceleration state. 
The results show that compared with the fuzzy control system and PID control system, the fuzzy control system based on information fusion particle swarm optimization algorithm is more prominent for the motor speed fusion and control ability under the acceleration condition, which mainly shows that the motor speed has been stable without significant floating phenomenon.

However, the condition fusion and control phenomenon of the other two algorithms are relatively poor, and the motor speed changes obviously.

It can be seen that the fuzzy control system based on the information fusion particle swarm optimization algorithm is stable for the fusion and control ability of the acceleration state of the electric vehicle.

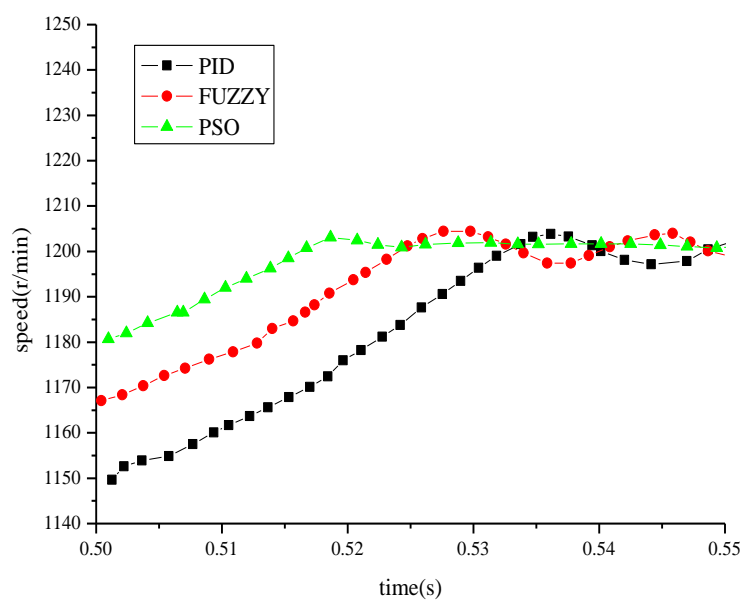

Figure 8: Speed comparison curve of power motor of new energy vehicle during acceleration

The speed comparison curve of the power motor of new energy vehicle when it decelerates under the working condition is shown in Figure 9.

From the data and trend chart in the figure, it can be seen that under the effect of three different algorithms, the speed of electric vehicle motor under the deceleration condition shows different situations.

Based on the information fusion particle swarm optimization, the fuzzy control system has the strongest ability of condition fusion and control, and achieves the stability of motor speed in the shortest time.

The other two algorithms cannot effectively achieve the motor fusion and control under the deceleration condition, and there is a relatively obvious speed instability phenomenon.

It can be seen that the fuzzy control system based on the information fusion particle swarm optimization algorithm has a significant ability for the fusion and control of the working condition of the electric vehicle in the deceleration state.

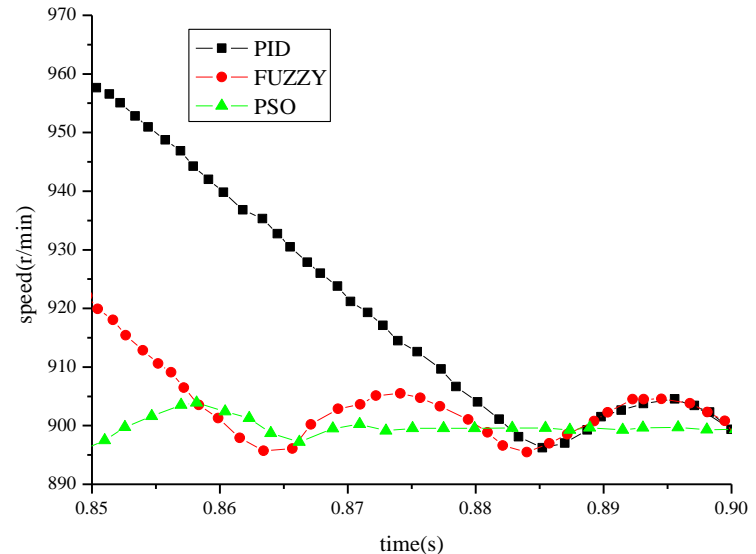

Figure 9: Speed comparison curve of power motor of new energy vehicle during deceleration

\section{Conclusions}

In this study, through the construction of fuzzy control system based on information fusion particle swarm optimization algorithm, the ability of information fusion particle swarm optimization algorithm, fuzzy control system and PID control system for motor condition fusion and control is studied in the three states of acceleration, deceleration and stability.

The results show that, compared with the fuzzy control system and PID control system, the fuzzy control system based on information fusion particle swarm optimization algorithm has outstanding ability for the fusion and control of electric vehicle motor in stable state, acceleration state and deceleration state, and the motor speed stability is very high without obvious fluctuation.

The motor greatly reduces the loss of energy and improves the efficiency of energy use. It can be seen that the algorithm model in this study has a significant ability of adaptive fusion control for new energy vehicles. There are also some deficiencies in the research process of this study, mainly in the conclusion of the research on the working condition of electric vehicle motor is more out of the experimental and theoretical stage, there will be many factors and problems for the actual field operation. Because many external factors are ignored in the experimental stage, the results are not convincing. However, from the qualitative point of view, this study provides a valuable reference for the control of the motor condition of new energy vehicles.

\section{References}

[1] Jiang G., He H., Yan J, et al. (2018) Multiscale convolutional neural networks for fault diagnosis of wind turbine gearbox. IEEE Transactions on Industrial Electronics, 66(4), 3196-3207. 
[2] Zhou D., Al-Durra A., Gao F, et al. (2017) Online energy management strategy of fuel cell hybrid electric vehicles based on data fusion approach. Journal of power sources, 366, 278-291.

[3] Zhang H., Wang J. (2016) Adaptive sliding-mode observer design for a selective catalytic reduction system of ground-vehicle diesel engines. IEEE/ASME Transactions on Mechatronics, 21(4), 2027-2038.

[4] Yi Z., Bauer P. H. (2017) Adaptive multiresolution energy consumption prediction for electric vehicles. IEEE Transactions on Vehicular Technology, 66(11), 10515-10525.

[5] Zhou D., Ravey A., Al-Durra A, et al. (2017) A comparative study of extremum seeking methods applied to online energy management strategy of fuel cell hybrid electric vehicles. Energy conversion and management, 151, 778790.

[6] Mahmoud M. S., Alyazidi N. M., Abouheaf M. I. (2017) Adaptive intelligent techniques for microgrid control systems: A survey. International Journal of Electrical Power \& Energy Systems, 90, 292-305.

[7] Enang W., Bannister C. (2017) Modelling and control of hybrid electric vehicles (A comprehensive review). Renewable and Sustainable Energy Reviews, 74, 1210-1239.

[8] Yin D., Sun N., Shan D, et al. (2017) A multiple data fusion approach to wheel slip control for decentralized electric vehicles. Energies, 10(4), 461.

[9] Zhu Q., Dai B., Huang Z, et al. (2017) An adaptive longitudinal control method for autonomous follow driving based on neural dynamic programming and internal model structure. International Journal of Advanced Robotic Systems, 14(6), 1729881417740711.
[10] Wu D., Li Y., Du C, et al. (2018) Fast velocity trajectory planning and control algorithm of intelligent 4WD electric vehicle for energy saving using time-based MPC. IET Intelligent Transport Systems, 13(1), 153-159.

[11] Rind S. J., Ren Y., $\mathrm{Hu}$ Y, et al. (2017) Configurations and control of traction motors for electric vehicles: A review. Chinese Journal of Electrical Engineering, 3(3), 1-17.

[12] Traub M., Maier A., Barbehön K. L. (2017) Future automotive architecture and the impact of IT trends. IEEE Software, 34(3), 27-32.

[13] Zhao B., Xu N., Chen H, et al. (2019) Stability control of electric vehicles with in-wheel motors by considering tire slip energy. Mechanical Systems and Signal Processing, 118, 340-359.

[14] Zhou D., Al-Durra A., Matraji I, et al. (2018) Online energy management strategy of fuel cell hybrid electric vehicles: a fractional-order extremum seeking method. IEEE Transactions on Industrial Electronics, 65(8), 6787-6799.

[15] Jing L., Wang T., Zhao M, et al. (2017) An adaptive multi-sensor data fusion method based on deep convolutional neural networks for fault diagnosis of planetary gearbox. Sensors, 17(2), 414.

[16] $\mathrm{Hu}$ Y., Li W., Xu K, et al. (2018) Energy management strategy for a hybrid electric vehicle based on deep reinforcement learning. Applied Sciences, 8(2), 187.

[17] Chen T., Xu X., Chen L, et al. (2018) Estimation of longitudinal force, lateral vehicle speed and yaw rate for four-wheel independent driven electric vehicles. Mechanical Systems and Signal Processing, 101, 377-388. 